\title{
Implantation of Mouse Embryos in the Rat Uterus
}

\author{
Hirotada Tsujir, Tetsuya SHigeTA \\ and Motokazu Yoshida \\ Faculty of Agriculture, Shinshu University, \\ Minamiminowa-mura, Nagano-ken 399-45
}

(Received March 24, 1980)

TARKOWSKI ${ }^{1)}$ has conducted experiments with interspecies mammalian egg transfer between rats and mice. He observed that rat blastocyst formed egg-cylinder structure when it was transferred to mice, but mouse blastocyst did not differentiate in the inner cell mass after transfer into rat uterus. The present experiment was executed to examine the possibility of implantation of mouse blastocysts after transfer into rat uterus.

\section{Materials and Methods}

Mature female mice of the ICR strain (8 weeks old) were used as donors of blastocysts, and pseudopregnant female rats of the Wistar strain ( 3 months old) were used as recipients. The animals were kept in the room under controlled temperature (ca. $20^{\circ} \mathrm{C}$ ) and light (12 hr light and $12 \mathrm{hr}$ dark). Vaginal smears were examined daily for 2 weeks before the experiment. On the day of proestrous, the female mice were caged with adult male mice. Pseudopregnant female rats were prepared by mating with vasectomized male rats. Observation of vaginal plug was estimated to be day 1 of pregnancy or pseudopregnancy. The donor mice were killed on day 4 of pregnancy. The medium used in the experiment was Krebs-Ringer bicarbonate solution ( $\mathrm{pH}$ 7.4). The embryos were collected by flushing the uterine horns. The blastocysts collected in a watch glass were examined under microscope, and normal blastocysts were used for the transfer. The recipient rats were operated on day 5 of pseudopregnancy under anaesthesia with ether. Usually five or six blastocysts were transferred into each uterine horn at the portion of utero-tubal junction. Recipient animals were killed 2 to 4 days after transfer and their uteri were removed. The uteri were fixed in Bouin fixatives, and preparations sectioned at $8 \mu$ and stained with haematoxylin and eosin were prepared for examination of implantation sites.

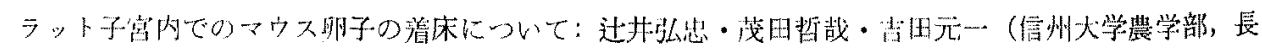

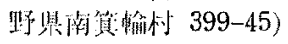




\section{Results and Discussion}

In total, 101 mouse blastocysts were transferred into the uteri of 9 rats, and 37 blastocysts $(37 \%)$ formed the implantation sites. The word "implantation" denotes decidual swelling regardless of the presence or absence of a living blastocyst.

On day 7 of pseudopregnancy ( 2 days after transfer), 12 of 23 transferred blastocysts $(52 \%)$ formed the implantation sites where mouse embryos were found in the uterine crypts. But the majority of them had not been in contact with epithelium. These embryos had no blastocoele which would have been seen in normal embryos, and might have become morphologically abnormal after transfer.

On day 8 of pseudopregnancy ( 3 days after transfer), 20 of 45 transferred blastocysts (44\%) formed the implantation sites, but they were dead except for one embryo which had implanted normally in the rat uterus (Plate 1). The trophoblast of the implanted embryo was closely connected with mucosa, and the epithelium had been broken down at the implantation site. The inner cell mass hung down into the yolk cavity, and egg-cylinder had been formed. A cavity which appeared to be the proamniotic cavity was found in the center of the egg-cylinder. The ectoplacental cone was found. The endoderm and ectoderm could not be distinguished, while these were clearly seen in the embryos of day 6 normal pregnant mice. The embryo was almost morphologically normal, but its developmental stage was slightly delayed in comparison with that of the normal pregnant mouse. The size of the embryo was $144 \mu \times 48 \mu$ and this was smaller than the average size of the embryo of day 6 of the normal pregnant mice $(180.6 \mu \times 61.6 \mu)$.

TARKOWSKI $^{1)}$ and other workers ${ }^{2-6)}$ described that the egg-cylinders were small and incomplete when rat eggs were transferred into the mouse genital tracts; their find-

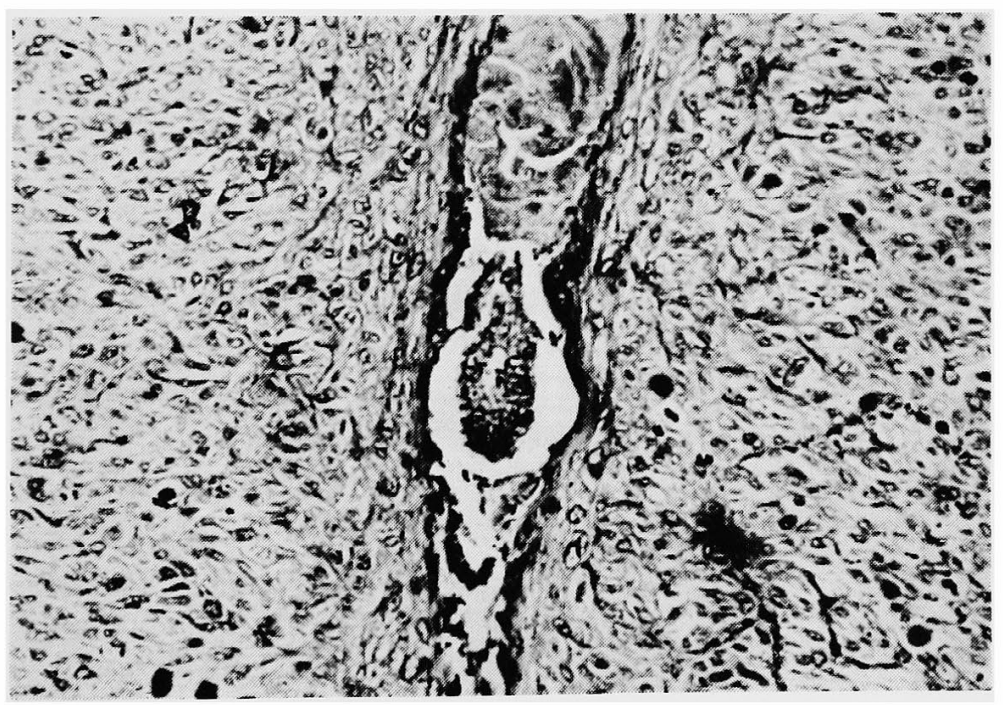

Plate 1. Implantation site of mouse blastocyst in the rat uterus. Complete implantation was observed and the egg-cylinder can be seen. $(\times 100)$. 
ings are agreeable with the present result.

After 9 days of pseudopregnancy, no living embryos were found in any of the implantation sites. This indicates that mouse eggs might be capable of implantation and formation of egg-cylinder in the rat uterus, but further development of the implanted embryo would be difficult. The failure in the successful implantation of mouse eggs in the rat uterus might be due to the ultrastructural unconformity of the cell surface $^{2)}$, immunological incompatibility ${ }^{5)}$ and inadequate hormone balance ${ }^{5)}$.

\section{References}

1) Tarkowski, A. K., J. Embryol. exp. Morph., 10: 476-495. 1962.

2) Potts, D. M., I. B. Wilson and M. S. R. Smith, J. Reprod. Fert., 22: 425-428. 1970.

3) Rossant, J., J. Embryol. exp. Morph., 36: 163-174. 1976.

4) Copp, A. J. and J. Rossant, J. Reprod. Fert., 52: 119-121. 1978.

5) HAkansson, S., Ö, Lundovist and B. O. Nilsson, Int. J. Fertil., 23: 148-151. 1978.

6) TACHI, S. and C. TACHI, Develop. Biol, 68: 203-223. 1979. 